Fukushima J. Med. Sci.,

Vol. 61, No. 1, 2015

[Original Article]

\title{
LONG-TERM FOLLOW-UP OF FREE VASCULARIZED FIBULAR HEAD GRAFT FOR RECONSTRUCTION OF THE PROXIMAL HUMERUS AFTER WIDE RESECTION FOR BONE SARCOMA
}

\author{
SOICHI EJIRI, TAKAHIRO TAJINO, RYOICHI KAWAKAMI, \\ MICHIYUKI HAKOZAKI and SHIN-ICHI KONNO \\ Department of Orthopaedic Surgery, Fukushima Medical University School of Medicine
}

(Received January 27, 2015, accepted March 10, 2015)

\begin{abstract}
Purpose : This study investigated the shape of bone grafts and associations with upper limb function over the long term after free vascularized fibular head graft (FVFHG) for reconstruction of the proximal humerus after wide resection for bone sarcoma.

Methods : Patients comprised 3 women who had undergone FVFHG at least 5 years previously. Age at surgery was 12 years in 2 cases and 76 years in one. The mean follow-up periods were 10 years 4 months. Evaluated parameters comprised : 1) graft hypertrophy, and 2) shape of the fibular head as changes in shape of the bone graft ; and 3) ISOLS score, and 4) DASH score as indicators of upper limb function.

Results : Rates of graft hypertrophy of the fibular shaft were $-14 \%,-17 \%$, and $-20 \%$, respectively, with transverse diameter decreasing in all cases. In terms of changes in shape of the grafted fibular head, transverse diameter had diminished in 2 patients $(-5 \mathrm{~mm}$ and $-2 \mathrm{~mm}$ ), and the head had been completely resorbed in the remaining patient. Both patients in whom the fibular head remained were young, and both had good ISOLS scores $>80 \%$ and good DASH scores of 5.0 and 8.3. The patient in whom the fibular head had been resorbed was elderly, with ISOLS and DASH scores of $73.3 \%$ and 34.2 , respectively ; comparatively poor compared with the other two. A comparison of ISOLS and DASH scores before and after fibular head resorption, however, showed no deterioration in either score.

Conclusions : Long-term follow-up of humerus reconstruction by FVFHG showed no deterioration in upper limb function despite the risk of fibular head resorption. FVFHG of the proximal humerus is a reconstruction technique that can provide good long-term upper limb function.
\end{abstract}

Key words : malignant humeral tumor, proximal humerus reconstruction, free vascularized fibular head graft, graft deformity, upper limb function

\section{INTRODUCTION}

Achieving excellent upper limb function after resection of the proximal humerus for malignant bone tumor requires the use of a reconstruction technique capable both of conserving function as a spacer over the long term as well as maintaining shoulder joint stability and good elbow joint function. Previous methods have included replacement with prostheses such as prosthetic joints for use in treating tumors ${ }^{1-3)}$, recycle bone treated in various ways (by Pasteurization ${ }^{3)}$, radiation ${ }^{4}$, or liquid nitrogen freezing ${ }^{5)}$ ), allograft ${ }^{1)}$, and autologous bone grafting with microsurgical technique. Among these, the microsurgical technique of free vascularized fibular head grafting (FVFHG) not only enables preservation of shoulder function, but also has the advantages of a good rate of bone-union and a low

Corresponding author : Soichi Ejiri, MD, PhD E-mail : sejiri@fmu.ac.jp

https://www.jstage.jst.go.jp/browse/fms http://www.fmu.ac.jp/home/lib/F-igaku/ 
infection rate, and excellent results from this method have been reported ${ }^{6,7)}$. As yet, however, no studies have reported changes in the shape of bone grafts more than 5 years after FVFHG, nor have their effect on upper limb function been investigated in detail. This study investigated shape of the bone graft and associations with upper limb function in 3 patients who had undergone reconstruction of the proximal humerus by FVFHG at least 5 years previously.

\section{PATIENTS AND METHODS}

Patients were 3 women who had undergone proximal humerus reconstruction using FVFHG after malignant bone tumor resection in our department at least 5 years previously (Table 1). Age at surgery was 12 years in two cases and 76 years in one. Histopathological diagnosis for the two young patients was osteosarcoma, and that for the elderly patient was chondrosarcoma. In terms of the Musculoskeletal Tumor Society (MSTS)/International Society of Limb Salvage (ISOLS) surgical staging system (revised according to Enneking's classifica$\operatorname{tion}^{8)}$ ), one patient was Stage IB (low grade (G1), extracompartmental lesion (T2), without metastasis (M0)) and the other two were Stage IIB (high grade (G2), extracompartmental lesion (T2), without metastasis (M0)).

Tumor resection comprised wide resection in all cases. In terms of the extent of osteotomy, type $\mathrm{IB}^{9)}$ resection (intra-articular resection of the humerus alone) was performed in cases 1 and 3 those were not invaded intra-articularly, and type $\mathrm{VB}^{9)}$ resection (extra-articular resection including the acetabular roof and distal clavicle) in case 2 that was invaded intra-articularly.

Reconstruction was performed in all cases with FVFHG following the sling procedure ${ }^{6)}$ (Fig. 1-3), in which the palmaris longs tendon and/or biceps femoris tendon maintaining the continuity of fibular head are attached to the scapula for the purpose of preserving scapulohumeral flexibility. Since the fibular graft was long (21-25 cm ; mean, $23 \mathrm{~cm})$, the pero- neal artery and vein were used as vascular pedicle, and were attached to the deep brachial artery and vein, respectively. In all cases, peroneal artery perforator island skin flap was prepared and used for blood flow monitoring. In the two patients who underwent intra-articular resection, the proximal end of the bone graft was secured by suturing the biceps femoris tendon attached to the bone graft to the biceps brachii tendon, and the tibiofibular joint capsule to the shoulder joint capsule. In the patient who underwent extra-articular resection, the biceps femoris tendon was secured to the clavicle. The distal end of the bone graft was secured to the shaft of the

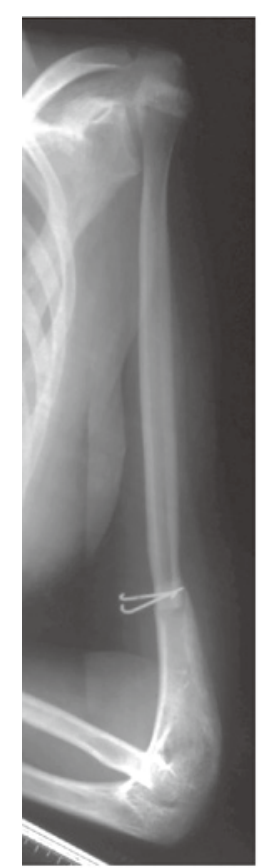

Fig. 1a

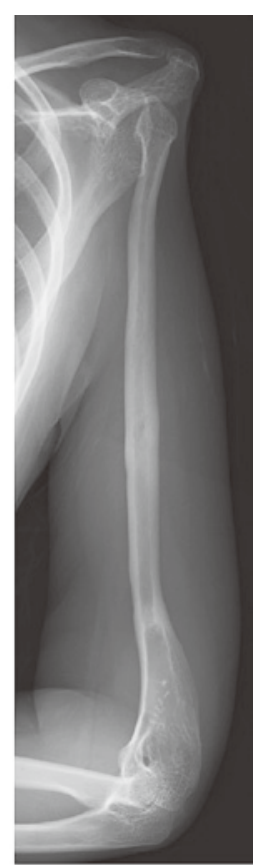

Fig. 1b
Fig. 1. Case 1: Plain radiographs immediately after surgery(a) and 15 years 6 months postoperatively(b) a. The patient had undergone intra-articular resection, and the proximal end of the graft was secured by suturing the biceps femoris tendon to the biceps brachii tendon, and the tibiofibular joint capsule to the shoulder joint capsule. The distal end was secured with Kirschner wires.

b. Transverse diameters of both the bone shaft of the fibular graft and of the fibular head have diminished.

Table 1. Patients' data

\begin{tabular}{cccccccc}
\hline Case & Age(y) / Gender & Diagnosis & $\begin{array}{c}\text { Surgical } \\
\text { staging }^{8)}\end{array}$ & $\begin{array}{c}\text { Type of } \\
\text { resection }\end{array}$ & $\begin{array}{c}\text { Graft length } \\
(\mathrm{cm})\end{array}$ & $\begin{array}{c}\text { Vascular } \\
\text { pedicle }\end{array}$ & $\begin{array}{c}\text { Follow-up } \\
\text { period }\end{array}$ \\
\hline 1 & $12 \mathrm{~F}$ & Osteosarcoma & IIB & IB & 21 & PAV* $^{*}$ & $15 \mathrm{y} 6 \mathrm{~m}$ \\
2 & $12 \mathrm{~F}$ & Osteosarcoma & IIB & VB & 25 & PAV* $^{*}$ & $9 \mathrm{y} 10 \mathrm{~m}$ \\
3 & $76 \mathrm{~F}$ & Chondrosarcoma & IB & IB & 23 & PAV* $^{*}$ & $6 \mathrm{y}$ \\
\hline
\end{tabular}




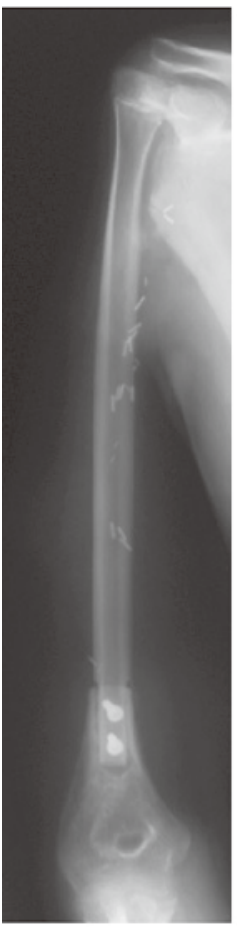

Fig. 2a

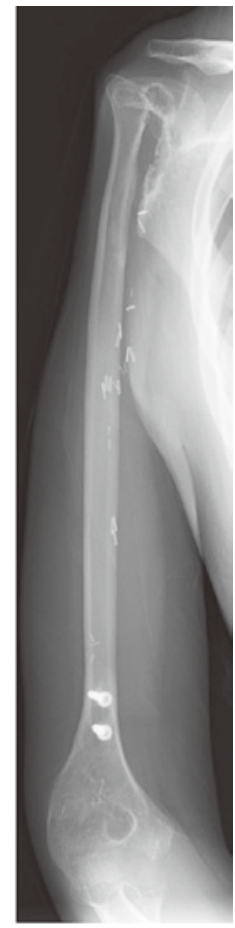

Fig. 2b
Fig. 2. Case 2 : Plain radiographs immediately after surgery(a) and 9 years 10 months postoperatively(b) a. The patient had undergone extra-articular resection, and the proximal end of the graft was secured by attaching the biceps femoris tendon to the clavicle. The distal end was secured with screws.

b. Transverse diameters of both the bone shaft of the fibular graft and of the fibular head have diminished.

humerus with Kirschner wires and screws in two patients, and in the vicinity of the elbow in one patient, with a plate used in that case to improve stability.

Postoperative therapy comprised use of a sling by all patients until bone-union of the distal end of the bone graft had been achieved, as well as active and passive wrist and finger exercises and with isometric exercises of the muscles around the shoulder and elbow. After bone-union had been achieved, the sling was removed, and the patient started to engage proactively in active and passive shoulder and elbow exercises.

The monitoring flap showed good blood supply, and the bone grafts successfully engrafted in all three patients. In all cases, 3 months was required for postoperative bone-union of the distal end of the bone graft. No fracture or infection of the fibular graft was encountered. Post-reconstruction follow-up periods for these three patients were 15 years 6 months, 9 years 10 months, and 6 years.

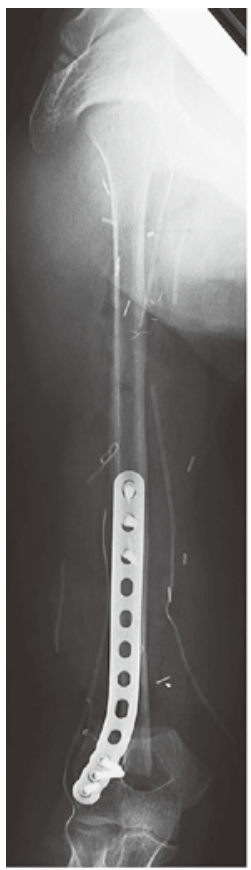

Fig. 3a

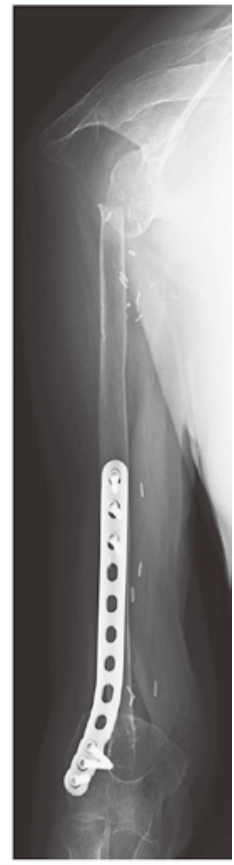

Fig. 3b
Fig. 3. Case 3 : Plain radiographs immediately after surgery(a) and 6 years postoperatively(b)

a. The patient had undergone intra-articular resection, and the proximal end of the graft was secured by suturing the biceps femoris tendon to the biceps brachii tendon, and the tibiofibular joint capsule to the shoulder joint capsule. The distal end was secured with a plate because it was near the elbow joint.

b. Transverse diameter of the bone shaft of the fibular graft has diminished, and the fibular head has been completely resorbed.

We evaluated changes in shape of the fibular graft and upper limb function in all three patients. Changes in shape of the fibular graft were evaluated using plain radiographs taken immediately postoperatively and at final follow-up examination to compare graft hypertrophy of the fibular graft and shape of the fibular head. The point at which graft hypertrophy was measured was determined by measuring diameter of the bone shaft other than in portions $5 \mathrm{~cm}$ from both ends of the fibular graft, and using the point where the change in transverse diameter over time was greatest. The rate of graft hypertrophy was calculated as a percentage according to the formula $((\mathrm{B}-\mathrm{A}) / \mathrm{A}) \times 100$, where $\mathrm{A}$ was the maximum transverse diameter immediately postoperatively and $B$ was the maximum transverse diameter at final follow-up. Shape of the fibular head was evaluated in terms of the change in maximum transverse diameter, as well as whether bone resorption had occurred. These values were measured three times 
by the same investigator (S.E.), and mean values were used.

Upper limb function was evaluated in terms of two different assessment forms, Enneking's affected upper limb functional evaluation (ISOLS score) ${ }^{10)}$ and the Disabilities of the Arm, Shoulder, and Hand (DASH) score ${ }^{11)}$ at the final follow-up examination. Associations with changes in shape of the fibular graft were also investigated in the same time.

ISOLS score is a representative assessment form for evaluating residual function of the affected limb after the tumor resection. This system evaluates the function of affected limb only. In ISOLS score, higher overall rating (\%) out of 30 points means better limb function. On the other hand, DASH score is an assessment form for evaluation of overall physical function and symptoms in patients with several musculoskeletal disorders. In this system, the general upper limb function including both affected and non-affected limbs is evaluated. In DASH score, higher point means severer dysfunction of bilateral upper limbs.

\section{RESULTS}

Changes in shape of the fibular graft (Table 2, Figs. 1-3)

1. Graft hypertrophy

Graft hypertrophy was $-17 \%$ in Case $1,-20 \%$ in Case 2, and $-14 \%$ in Case 3, with transverse diameter diminishing in all cases.

2. Shape of the fibular head

Maximum transverse diameter of the fibular head decreased over time by $5 \mathrm{~mm}$ in Case 1 and by $2 \mathrm{~mm}$ in Case 2. In Case 3, the fibular head was completely resorbed.

\section{Upper limb function}

3. ISOLS score $^{10)}$ (Table 3a)

ISOLS score in both Cases 1 and 2 was $80 \%$, but was only $73 \%$ in Case 3 (the elderly patient with resorption of the fibular head), comparatively lower than in the other two.

4. DASH score ${ }^{11)}$ (Table $3 b$ )

DASH scores were 8.3 for Case 1 and 5 for Case 2 , both representing good scores. In Case 3 (the elderly patient with resorption of the fibular head), DASH score was 34.2, comparatively poor compared with the other two.

Table 2. Changes in shape of the fibular graft

\begin{tabular}{ccc}
\hline Case & Graft hypertrophy* $(\%)$ & Head diameter**(mm) \\
\hline 1 & -17 & -5 \\
2 & -20 & -2 \\
3 & -14 & resorption \\
\hline
\end{tabular}

* Increase in transverse diameter of the shaft of the fibular graft

** Change in maximum diameter of the fibular head

a. ISOLS score ${ }^{10)}$

Table 3. Upper limb function

\begin{tabular}{cccccccc}
\hline & \multicolumn{7}{c}{ Functional evaluation } \\
\cline { 2 - 8 } Case & Pain & Function & $\begin{array}{c}\text { Emotional } \\
\text { acceptance }\end{array}$ & $\begin{array}{c}\text { Positioning of } \\
\text { the hand }\end{array}$ & $\begin{array}{c}\text { Manual } \\
\text { dexterity }\end{array}$ & $\begin{array}{c}\text { Lifting } \\
\text { ability }\end{array}$ & $\begin{array}{c}\text { Overall rating } \\
(\%)\end{array}$ \\
\hline 1 & 5 & 4 & 3 & 3 & 5 & 4 & 80 \\
2 & 5 & 4 & 3 & 3 & 5 & 4 & 80 \\
3 & 5 & 3 & 3 & 3 & 5 & 3 & 73 \\
\hline
\end{tabular}

b. DASH score ${ }^{11)}$

\begin{tabular}{cccc}
\hline Case & Disability/Symptom & Sports/Music & Work \\
\hline 1 & 8.3 & 12.5 & 0 \\
2 & 5.0 & - & 0 \\
3 & 34.2 & - & - \\
\hline
\end{tabular}


Table 4. Associations between change in shape of the fibular graft and upper limb function in case 3

\begin{tabular}{cccc}
\hline Follow-up period & Resorption of the fibular head & ISOLS score $^{10)}(\%)$ & DASH score $^{11)}\left(\right.$ points $^{2}$ \\
\hline 0 y $4 \mathrm{~m}$ & no & 70 & 57.3 \\
0 y $8 \mathrm{~m}$ & yes & 70 & 34.2 \\
6 y & yes & 73 & 34.2 \\
\hline
\end{tabular}

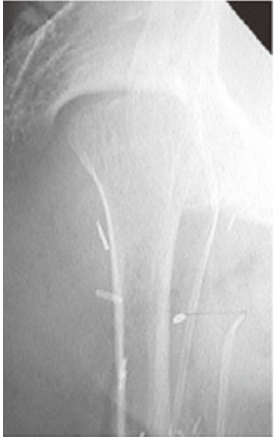

Fig. 4a

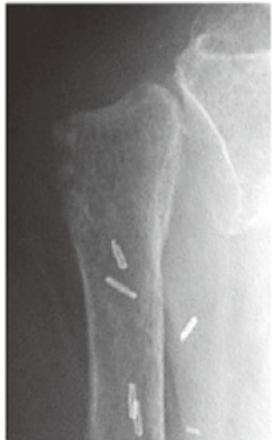

Fig. 4b

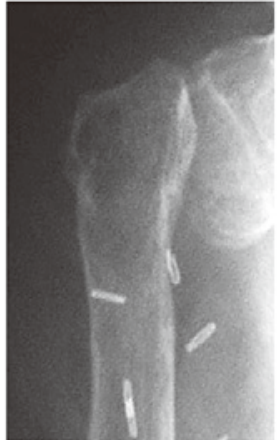

Fig. 4c

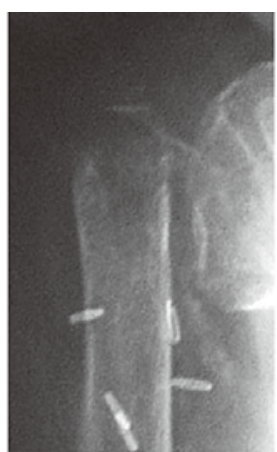

Fig. 4d

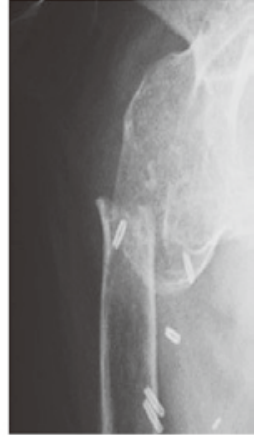

Fig. 4e

Fig. 4. Changing shape of the fibular head in case 3

Plain radiographs immediately after surgery(a), 4 months(b), 6 months(c), 8 months(d), and 6 years postoperatively(e). The fibular head was completely resorbed between 6 and 8 months postoperatively.

Associations between change in shape of the fibular graft and upper limb function (Table 4, Fig. 4a-e)

To ascertain the effect of changes in shape of the fibular graft on upper limb function, we compared ISOLS and DASH scores in Case 3 (the elderly patient with resorption of the fibular head) before and after resorption occurred. Four months postoperatively, before resorption was evident, respective scores were $70 \%$ and 57.3 , and 8 months postoperatively, immediately after resorption had occurred, these values were $70 \%$ and 34.2. At final follow-up examination 6 years postoperatively, scores were $73 \%$ and 34.2 , with upper limb function having improved over time.

\section{DISCUSSION}

Hand function is frequently preserved after proximal humerus resection for malignant tumor. To achieve good upper limb function, supplementing a wide-ranging defect with some sort of spacer, and conservation of shoulder joint stability and elbow joint function is therefore important. A number of different reconstructive methods to enable such outcomes have been reported in recent years. Prosthetic joints for use in treating tumors have the advantages of not requiring the sacrifice of autologous tissue and of providing excellent early postoperative support, and reliable results have been reported from this method ${ }^{1,2)}$. However, some risks are en- tailed, including deep infection as well as long-term loosening or fracture of the junction. Recent years have also seen reports of reconstruction using bone treated with Pasteurization ${ }^{3)}$, radiation ${ }^{4}$, or liquid nitrogen freezing ${ }^{5)}$, as well as allografts ${ }^{1)}$. Reported risks, however, include fracture, non-union, bone resorption, and infection, and allografts are difficult to obtain in Japan. According to the latest systematic review $^{1)}$, the reported incidence of complications is 4.5-8.5\% for prosthetic joints and $0-150 \%$ for allografts, with some studies finding an extremely high rate. Reconstruction using $\mathrm{FVFHG}^{6,7)}$ has the disadvantages of requiring a long operating time and a high level of skill in performing microsurgery, as well as the sacrifice autologous tissue, but as living bone is utilizes, this approach promises to provide an excellent method of reconstruction in terms of a high bone-union rate, ensuring long-term strength and a low rate of infection. In fact, bone-union was achieved within 3 months after surgery in all three of our patients, with no occurrence of infection or fracture, and the graft maintained its role as a good spacer over the long term. This suggests the superiority of surgical techniques using living bone.

Graft hypertrophy represents a well-known long-term change in the shape of the fibular graft. This did not occur in any of our patients, and transverse diameter of the bone shaft actually diminished. This may have been due to the contribution of mechanical loading. De Boer et al. ${ }^{12)}$ studied 62 patients who underwent free vascularized fibula 
grafting into the long bones of the limbs, and found that graft hypertrophy occurred in $43 \%$ of grafts in the legs, but only $20 \%$ of those in the arms. Wada et $a l .{ }^{13)}$ reported that among 12 patients who underwent arthrodesis with free vascularized fibula grafting after malignant bone tumor resection near the knee, graft hypertrophy did not occur in those who had undergone firm immobilization with the additional use of internal fixation materials. This suggests that mechanical loading is a major factor contributing to graft hypertrophy. The reason for the decrease in transverse diameter seen in our patients may have been that unlike the legs, these grafts were not subject to sustained loading. A further contributory factor in our cases may have been that as one of the patients was an office worker and the other two were young women who were not yet working, they were not required to perform heavy labor, and because the sling procedure leaves the shoulder mobile, this meant that the load was distributed.

The resorption of the fibular head seen in one of our patients may have been caused by inadequate perfusion as a result of the choice of vascular pedicle. The fibular head is fed by the anterior tibial artery, so when the peroneal artery is used as the vascular pedicle, as in our patients, it is possible that perfusion of the fibular head was insufficient and bone resorption therefore occurred. In a study of fresh cadavers, Taylor $e t$ al. $^{14)}$ found that if the anterior tibial artery is used as the vascular pedicle perfusion can be maintained to the fibular head and the proximal two-thirds of the fibula, suggesting that using the anterior tibial artery for the vascular pedicle may be helpful in preventing resorption of the fibular head. However, Onoda et al. ${ }^{7}$ reported that distal graft fracture and non-union occurred in two of four patients who underwent proximal humeral reconstruction by FVFHG with the anterior tibial artery as the pedicle, and suggested as the cause that if the anterior tibial artery is used as the pedicle for long FVFHG, even if the graft is within the proximal two-thirds of the fibula, there is a risk of inadequate perfusion of the distal region. In long FVFHG, as used in our patients, using the peroneal artery as the pedicle thus entails a risk of femoral head resorption, but use of the anterior tibial artery conversely increases the risk of fracture and pseudarthrosis in the distal region. Ad-El et al. ${ }^{15)}$ therefore recommended reconstruction with a bipedicle that uses both the fibular head feeding branch of the anterior tibial artery and the peroneal artery. This entails problems including increased complexity of the procedure, prolonged operating time, and the need to secure recipient vessels, but is nevertheless a method that may be tried in some patients.

We compared postoperative upper limb function as evaluated by ISOLS score with that for other surgical procedures. A systematic review by Teunis $e t$ $a l .{ }^{1}$ that summarized reports of prosthetic joint replacement and allografting in the proximal humerus found that reported scores for prosthetic joint replacement ranged from $61 \%$ to $77 \%$, and those for allografting ranged from $50 \%$ to $78 \%$. Our patients thus had good upper limb function as evaluated by ISOLS score compared with those who underwent other surgical procedures.

An examination of scores for the different items that make up the ISOLS score revealed that all our patients exhibited good scores for pain and manual dexterity. In terms of emotional acceptance and positioning of the hand, however, they all had low scores. The reason for the low scores on emotional acceptance may have been that the two young patients made high demands with respect to the cosmetic appearance of soft tissue defects, meaning that they may have had more problems with the extent of resection than with the method of reconstruction. For Case 3, the elderly patient, the main reason for dissatisfaction was probably that it became somewhat more difficult for her to support herself to compensate for leg weakness. All three patients scored low on positioning of hand because the deltoids and rotator cuff, which provide the motive force for raising the shoulder, had been removed, making it impossible for them to lift their hands above their shoulders in the frontal plane. The objective of this procedure, however, is for the bone graft to act as a spacer, enabling good shoulder stability and elbow flexion to be achieved. In fact, external and internal rotation by the shoulder girdle and active elbow movements provided good arm control, with comparatively good results for function and lifting ability.

Both of the young patients showed extremely high DASH scores. One possible reason for this may have been that DASH score comprises a functional evaluation of both upper limbs. If good shoulder stability and elbow function can be preserved by means of a fibular graft, patients can perform almost all actions involved in daily living with the healthy arm, assisted by the affected arm. In fact, shoulder contracture of the healthy side was evident in Case 3, and scores for overhead actions and heavy labor were lower than those of the other two patients. No cases with the use of DASH to 
evaluate proximal humerus reconstruction after tumor resection compared with other surgical procedures have been reported, and future studies are awaited.

In terms of the association between changes in shape of the fibular graft and upper limb function, upper limb function did not deteriorate after resorption even in Case 3, the patient with resorption of the fibular head. This means that as long as there is no great change in length and location of the fibula, the bone graft remains fully capable of fulfilling its role as a spacer even in the absence of the fibular head. In Case 3, the proximal end was secured by suturing the biceps femoris tendon to the biceps brachii tendon and the tibiofibular joint capsule to the shoulder joint capsule. Even at the final followup examination, by which time the fibular head had disappeared, reconstructed scaplohumeral function was maintained and no downward movement of the fibula was seen, with the proximal end of the fibula positioned in the area of the acetabular roof. It may be conjectured that this acted as a support point, preserving shoulder stability and elbow function and thus maintaining upper limb function.

As described above, although using the anterior tibial artery for the vascular pedicle reduces the risk of fibular head resorption, bone-union of the distal end can become problematic, and the surgical procedure becomes more complex when a double pedicle is used. Given, however, that there is little risk of functional deterioration even if the fibular head is resorbed, use of the peroneal artery as the vascular pedicle should not pose a problem for long FVFHGs.

\section{CONCLUSION}

We investigated long-term changes in shape of the fibular graft and upper limb function in patients who underwent proximal humerus reconstruction by FVFHG after wide resection. Although transverse diameter of the fibular graft decreased, no complications such as fracture and non-union were encountered, and good upper limb function was retained. Using the peroneal artery as the pedicle entails a risk of fibular head resorption, but this may have little effect on upper limb function. Although our study only involved three cases, the results suggest that proximal humerus reconstruction by FVFHG is an excellent surgical procedure capable of maintaining good upper limb function, and accumulation of information from further cases is anticipated.

\section{REFERENCES}

1. Teunis T, Nota SP, Hornicek FJ, et al. Outcome after reconstruction of the proximal humerus for tumor resection : a systematic review. Clin Orthop Relat Res, 472 : 2245-2253, 2014.

2. Cannon CP, Paraliticci GU, Lin PP, et al. Functional outcome following endoprosthetic reconstruction of the proximal humerus. J Shoulder Elbow Surg, 18 : 705-710, 2009.

3. Liu T, Zhang Q, Guo X, et al. Treatment and outcome of malignant bone tumors of the proximal humerus : biological versus endoprosthetic reconstruction. BMC Musculoskelet Disord, 15 : 69, 2014.

4. Kotb SZ, Mostafa MF. Recycling of extracorporeally irradiated autograft for malignant bone tumors : long-term follow-up. Ann Plast Surg, 71: 493-499, 2013.

5. Tsuchiya H, Wan SL, Sakayama K, et al. Reconstruction using an autograft containing tumour treated by liquid nitrogen. J Bone Joint Surg Br, 87 : 218-225, 2005.

6. Wada T, Usui M, Isu K, et al. Reconstruction and limb salvage after resection for malignant bone tumour of the proximal humerus. A sling procedure using a free vascularised fibular graft. J Bone Joint Surg Br, 81 : 808-813, 1999.

7. Onoda S, Sakuraba M, Asano T, et al. Use of vascularized free fibular head grafts for upper limb oncologic reconstruction. Plast Reconstr Surg, 127 : 1244-1253, 2011.

8. Enneking WF, Spanier SS, Goodman MA. A system for the surgical staging of musculoskeletal sarcoma. Clin Orthop, 153 : 106-120, 1980.

9. Malawer MM, Meller I, Dunham WK. A new surgical classification system for shoulder-girdle resections. Analysis of 38 patients. Clin Orthop Relat Res, 267 : 33-44, 1991.

10. Enneking WF, Dunham WK, Gebhardt MC, et al. A system of the functional evaluation of reconstructive procedures after surgical treatment of tumors of the musculoskeletal system. Clin Orthop Relat Res, 286 : 241-246, 1993.

11. Hudak PL, Amadio PC, Bombardier C. Development of an upper extremity outcome measure : the DASH (disabilities of the arm, shoulder and hand) [corrected]. The Upper Extremity Collaborative Group (UECG). Am J Ind Med, 29 : 602-608, 1996.

12. de Boer HH, Wood MB. Bone changes in the vascularised fibular graft. J Bone Joint Surg Br, 71 : 374-378, 1989.

13. Wada T, Usui M, Nagoya S, et al. Resection arthrodesis of the knee with a vascularised fibular graft. Medium- to long-term results. J Bone 
Joint Surg Br, 82 : 489-493, 2000.

14. Taylor GI, Wilson KR, Rees MD, et al. The anterior tibial vessels and their role in epiphyseal and diaphyseal transfer of the fibula : experimental study and clinical applications. Br J Plast Surg,
41 : 451-469, 1988.

15. Ad-El DD, Paizer A, Pidhortz C. Bipedicled vascularized fibula flap for proximal humerus defect in a child. Plast Reconstr Surg, 107 : 155-157, 2001. 